\title{
NUTRIÇÃO MINERAL DE MUDAS DE EUCALIPTO PRODUZIDAS SOB DIFERENTES LÂMINAS DE IRRIGAÇÃO E SUBSTRATOS ${ }^{(1)}$
}

\author{
Jane Luísa Wadas Lopes ${ }^{(2)}$, Iraê Amaral Guerrini ${ }^{(3)}$, João Carlos \\ Cury Saad ${ }^{(4)} \&$ Magali Ribeiro da Silva ${ }^{(5)}$
}

\begin{abstract}
RESUMO
Este trabalho teve como objetivo avaliar os efeitos das lâminas brutas de irrigação na concentração de nutrientes de mudas de Eucalyptus grandis, produzidas em diferentes substratos. O experimento foi realizado na Camará Mudas Florestais, em Ibaté, SP, e constituiu-se de um fatorial 5 x 4, sendo cinco lâminas brutas de irrigação diárias $(6,8,10,12$ e $14 \mathrm{~mm})$, aplicadas em diferentes horários (10, 13 e 16 h), e quatro substratos (FB - fibra de coco; CPV - casca de pinus e vermiculita; CATV - casca de pinus, carvão, turfa e vermiculita; e um MIX - $70 \%$ de CPV e 30 \% de FB). Foram determinadas os conteúdos dos nutrientes na parte aérea e no sistema radicular das mudas. Os resultados indicaram que houve influência das lâminas de irrigação e dos substratos no conteúdo dos nutrientes das mudas com maior acúmulo dos mesmos à medida que o fornecimento de água foi maior. As plantas crescidas nos substratos FB e CPV registraram os maiores acúmulos de nutrientes. CPV foi o melhor em se tratando do acúmulo dos macronutrientes nas raízes e também, juntamente com $\mathrm{FB}$, o melhor para o acúmulo dos micronutrientes nas raízes e na parte aérea. Não houve influência dos substratos no acúmulo dos macronutrientes na parte aérea, porém houve das lâminas de irrigação.
\end{abstract}

Termos de indexação: Eucalyptus grandis, viveiro, irrigação, nutrição mineral.

\footnotetext{
(1) Trabalho extraído da Tese de Mestrado do primeiro autor. Recebido para publicação em outubro de 2005 e aprovado em abril de 2007.

(2) Doutoranda em Agronomia-Irrigação e Drenagem, UNESP/FCA. Bolsista do CNPq. E-mail: jane.lopes@terra.com.br

(3) Professor Adjunto-Departamento de Recursos Naturais/Ciência do Solo. UNESP/FCA. Bolsista do CNPq. E-mail: iguerrini@fca.unesp.br

(4) Professor Adjunto-Departamento de Engenharia Rural. UNESP/FCA. E-mail: joaosaad@fca.unesp.br

(5) Professora Doutora-Departamento de Recursos Naturais/Ciências Florestais. UNESP/FCA. E-mail: magaliribeiro@fca.unesp.br
} 


\title{
SUMMARY: MINERAL NUTRITION OF EUCALYPT SEEDLINGS GROWN UNDER DIFFERENT IRRIGATION LEVELS AND SUBSTRATES
}

\begin{abstract}
The objective of this work was to evaluate the effects of gross irrigation depths on the nutrient concentration of Eucalyptus grandis seedlings, produced in different substrates. The experiment was carried out in Camará - Mudas Florestais, in Ibaté, São Paulo, in a factorial $5 \times 4$, with five daily irrigation levels $(6,8,10,12$ and $14 \mathrm{~mm})$, applied three times a day (at $10 \mathrm{am}, 13 \mathrm{pm}$ and $16 \mathrm{pm}$ ), on four substrates (FB-coconut fiber; $C P V$ - pine bark and vermiculite; $C A T V$ - pine bark, coal, turf and vermiculite and MIX- $70 \% C P V$ and $30 \%$ FB). The nutrient concentration was determined in the aerial part and roots of the seedlings, which was influenced by irrigation levels and substratum type. The nutrient concentrations rose with the increase of the irrigation levels. The nutrient accumulation was highest using $F B$ and $C P V$ substrates. $C P V$ was best for macronutrient accumulation and together with $F B$, best for micronutrient accumulation in roots and aerial parts. Macronutrient accumulation in the aerial part was influenced by the irrigation levels but not by the substratum.
\end{abstract}

Index terms: Eucalyptus grandis, tree nursery, irrigation, mineral nutrition.

\section{INTRODUÇÃO}

Substratos agrícolas são produtos usados como meio de crescimento de plantas (Brasil, 2004), sendo a estabilidade biológica fundamental, pois altas relações entre carbono e nitrogênio $(\mathrm{C} / \mathrm{N})$ podem levar à competição entre plantas e microrganismos por nutrientes, principalmente $\mathrm{N}$, fazendo com que as plantas, em conseqüência, apresentem sérias deficiências. Antes das fibras de coco surgirem como substrato, acreditava-se que as relações C/N de 18/1 para substratos eram altas (Gonçalves \& Poggiani, 1996). Atualmente sabe-se que as relações $\mathrm{C} / \mathrm{N}$ podem ser elevadas; trabalhos de Abad \& Nogueira (1998 citado por Martinez, 2002) e Lopes (2004), com relações C/N variando entre 74/1 e 194/1, não apresentaram problemas no desenvolvimento das mudas.

Devido à complexidade de substratos existentes, as recomendações nutricionais para mudas de eucalipto são dificultadas; no entanto, podem-se utilizar fertilizantes de liberação lenta (Barros et al., 1997) e outras formulações, observando que a faixa ideal de $\mathrm{pH}$ para absorção da maioria dos nutrientes é de 5,5 a 6,0 . As recomendações, na maioria das vezes, são feitas considerando os exames visuais de deficiência ou toxidez; não existem na literatura faixas de acúmulo para o sistema radicular e para a parte aérea (folhas, ramos e haste) das mudas que as qualifiquem como ideais nutricionalmente em cada estádio de desenvolvimento. Os acúmulos registrados em pesquisas, embora na sua grande maioria não estejam correlacionados ao manejo hídrico e aos substratos utilizados, variam muito de acordo com o manejo de viveiro e as condições ambientais, não possibilitando que se estabeleça uma faixa ideal, já que não foram verificados sintomas de toxidez e, ou, de deficiência (Lima et al., 1997; Higashi et al., 2000; Ismael, 2001; Silveira et al., 2001; Silva, 2003).
A principal finalidade da irrigação é proporcionar umidade necessária para o crescimento e desenvolvimento das plantas em menor período de tempo, pois a água representa cerca de $50 \%$ da biomassa fresca das espécies lenhosas (Kramer \& Boyer, 1995). As plantas, possuem controle sobre a perda de água, por meio da abertura e do fechamento dos estômatos. Estudos de eficiência de uso da água mostram que a produção de matéria seca total aumenta quando maior quantidade de água é usada (Alvarenga et al., 1994; Kramer \& Boyer, 1995; Sasse et al., 1996; Ismael, 2001; Silva, 2003; Lopes, 2004; Gruber, 2006). Silva (2003) verificou que os nutrientes nas plantas são influenciados pelo manejo hídrico aplicado às mudas; para a maioria dos nutrientes, os conteúdos foram maiores naquelas submetidas ao menor estresse hídrico. Lima et al. (1997) verificaram que a distribuição de nutrientes variou com o potencial de água no solo, o tipo de solo e a espécie de eucalipto.

Lopes (2006) aborda os diversos métodos que podem ser empregados para irrigar plantas em viveiros, mencionando o uso das barras de pulverização, melhorando significativamente a uniformidade de distribuição de água, aliada à economia significativa no consumo.

Este trabalho objetivou avaliar o acúmulo de nutrientes nas mudas de eucalipto submetidas a cinco lâminas brutas de irrigação diárias em quatro tipos de substrato.

\section{MATERIAL E MÉTODOS}

O experimento foi realizado de junho a outubro de 2003, na Camará - Mudas Florestais, no município de Ibaté, SP, cujas coordenadas geográficas são $21^{\circ} 56$ ' de latitude sul, $47^{\circ} 59$ ' de longitude oeste e aproximadamente $793 \mathrm{~m}$ de altitude. O clima da 
região é do tipo Cfa moderado chuvoso. Na região, a precipitação média mensal é de $116 \mathrm{~mm}$, oscilando entre 23 e $262 \mathrm{~mm}$ nos anos mais secos e mais úmidos, respectivamente.

As sementes usadas de Eucalyptus grandis Hill Ex. Maiden foram de um Pomar de Sementes Clonal (Boa Esperança do Sul - SP). Foram usados tubetes cilíndricos - cônicos de polietileno com quatro estrias internas salientes e capacidade em volume de $50 \mathrm{~cm}^{3}$. Como suporte, foram usadas, na casa de germinação, bandejas de polietileno com capacidade para 176 tubetes e, na área a céu aberto, mesas de estrutura metálica (teladas) com capacidade total de 1.240 tubetes. Na casa de germinação, os recipientes ocuparam $100 \%$ da capacidade da bandeja e, na área a céu aberto, $25 \%$ da área da mesa telada.

Foram testados os substratos comerciais: FB substrato de fibra de coco; CPV - substrato de casca de pinus e vermiculita; CATV - substrato de cascas de pinus, carvão, turfa e vermiculita; e um MIX (usado rotineiramente no viveiro da empresa) - $70 \%$ de CPV e $30 \%$ de FB.

$\mathrm{Na}$ adubação de base foram usados $4 \mathrm{~kg}$ do adubo de liberação lenta, formulação 15:10:10 (osmocote), para cada $\mathrm{m}^{3}$ de substrato. As adubações de cobertura, via água de irrigação (com lâmina bruta convencionada de $2 \mathrm{~mm}$ ), foram aplicadas na última passagem da barra pelo canteiro no final do dia. Na primeira adubação, aos 85 DAS - dias após a semeadura, foram usados: $3,0 \mathrm{~g} \mathrm{~L}^{-1}$ de nitrato de $\mathrm{Ca}, 1,0 \mathrm{~g} \mathrm{~L}^{-1}$ de sulfato de amônio, 1,0 $\mathrm{g} \mathrm{L}^{-1}$ de nitrato de $\mathrm{K}$ e 1,2 $\mathrm{g} \mathrm{L}^{-1}$ de sulfato de $\mathrm{Mg}$. Na segunda, aos $100 \mathrm{DAS}$, utilizaramse: $0,690 \mathrm{~g} \mathrm{~L}^{-1}$ de $\mathrm{K}_{2} \mathrm{O}$ e $0,418 \mathrm{~g} \mathrm{~L}^{-1}$ de $\mathrm{Ca}$, na forma de cloreto de potássio e cloreto de cálcio, respectivamente.

Aos 50 DAS foram separadas e medidas (dos grupos das médias e maiores) 1.600 mudas para compor a área útil e 680 mudas para compor a bordadura (dupla), com altura e diâmetros equivalentes, que foram alternadas, ocupando área de $49 \mathrm{~cm}^{2}$ cada uma.

A partir da experiência prática de Lopes (2004), utilizando microaspersão, por meio de uma barra de pulverização manual, foi definida a freqüência de irrigação em três horários ao longo do dia (10, 13 e $16 \mathrm{~h}$ ), com distribuição nos canteiros da seguinte maneira: $6 \mathrm{~mm}$ : $2,0 \mathrm{~mm}$ às $10 \mathrm{~h}, 13 \mathrm{~h}$ e $16 \mathrm{~h} ; 8 \mathrm{~mm}$ : $2,0 \mathrm{~mm}$ às $10 \mathrm{~h}$ e $16 \mathrm{~h}$ e $4 \mathrm{~mm}$ às $13 \mathrm{~h} ; 10 \mathrm{~mm}$ : $2,0 \mathrm{~mm}$ às $10 \mathrm{~h}$ e $4,0 \mathrm{~mm}$ às $13 \mathrm{~h}$ e $16 \mathrm{~h} ; 12 \mathrm{~mm}$ : $2,0 \mathrm{~mm}$ às $10 \mathrm{~h}, 4 \mathrm{~mm}$ às $13 \mathrm{~h}$ e $6 \mathrm{~mm}$ às $16 \mathrm{~h} ; 14 \mathrm{~mm}$ : $2,0 \mathrm{~mm}$ às $10 \mathrm{~h}$ e $6 \mathrm{~mm}$ às $13 \mathrm{~h}$ e $16 \mathrm{~h}$; o volume de água foi controlado pelo tempo de deslocamento da barra no canteiro e sua respectiva vazão (definidos em testes preliminares).

Para cada lâmina de irrigação foi destinado um canteiro no qual foram distribuídos quatro blocos ou repetições. Cada bloco continha os quatro substratos, cada um com 80 plantas úteis mais a bordadura correspondente.
Para caracterização química dos substratos foram feitas análises no Laboratório de Fertilizantes e Corretivos - Departamento de Recursos Naturais/ Ciência do Solo, da Faculdade de Ciências Agronômicas da UNESP, utilizando os métodos descritos pelo Ministério da Agricultura (Brasil, 1988). Para caracterização nutricional das plantas foram realizadas análises no Laboratório de Nutrição Mineral de Plantas do mesmo departamento.

Após 108 DAS, as mudas foram retiradas das embalagens e divididas em parte aérea e sistema radicular. O sistema radicular teve remoção completa do substrato e, assim como a parte aérea, foi submetido à secagem em estufa de ventilação forçada a $60^{\circ} \mathrm{C}$. Após esse procedimento, o material foi encaminhado ao laboratório, onde foram determinadas as concentrações dos macro e dos micronutrientes, segundo Malavolta et al. (1997).

\section{RESULTADOS E DISCUSSÃO}

\section{Características químicas dos substratos}

Analisando os teores registrados no quadro 1, verifica-se que $\mathrm{CPV}$ apresentou o maior teor de $\mathrm{N} \mathrm{e}$ FB o menor, e que o maior teor de $\mathrm{C}$ foi obtido para FB e o menor para CATV. Dessa maneira, verifica-se que, à exceção de CATV, todos os substratos apresentaram teores considerados adequados de matéria orgânica, segundo Abad et al. (1992) e Schmitz et al. (2002). Quanto à relação C:N, os valores obtidos são considerados altos para todos os substratos se comparados aos de Gonçalves \& Poggiani (1996), porém adequados quando comparados aos de Abad et al. (1992); a adubação de base e também a estabilidade biológica das fibras de coco pasteurizadas podem ter garantido que as mudas não tivessem apresentado sintomas visuais de deficiência e, ou, toxidez.

Com a definição da metodologia específica para substratos (Abreu, 2006), que deverá, provavelmente, estabelecer para $\mathrm{CE}$ e $\mathrm{pH}$ a mesma metodologia definida em 2003 pelo Comitê de Normatização Européia, não é possível comparar os valores obtidos com os recomendados até então para substratos, nem com a recomendação de Gonçalves et al. (2000), para produção de mudas de espécies florestais; contudo, os tratamentos utilizados nesta pesquisa não afetaram negativamente o desenvolvimento das mudas.

\section{Concentração de macronutrientes no sistema radicular e na parte aérea das mudas}

Analisando os resultados apresentados nos quadros 2 e 3 , verifica-se que, de modo geral, à medida que o fornecimento de água às mudas foi maior, maior foi o acúmulo de $\mathrm{N}, \mathrm{P}, \mathrm{K}, \mathrm{Ca}, \mathrm{Mg}$ e $\mathrm{S}$ no sistema radicular em todos os substratos. CPV foi o substrato que registrou os maiores acúmulos de todos os 
Quadro 1. Teores totais de nitrogênio, matéria orgânica, carbono, pH e condutividade elétrica no extrato $1: 2^{(1)}$ e teores disponíveis de macro e micronutrientes no extrato $1: 2^{(1)}$ nos substratos, antes da produção das mudas

\begin{tabular}{|c|c|c|c|c|}
\hline \multirow[b]{2}{*}{ Nutriente } & \multicolumn{4}{|c|}{ Substrato } \\
\hline & MIX & FB & CPV & CATV \\
\hline Nitrogênio $\left(\mathrm{g} \mathrm{kg}^{-1}\right)$ & 0,77 & 0,57 & 1,50 & 0,63 \\
\hline Matéria orgânica ( $\mathrm{g} \mathrm{kg}^{-1}$ ) & 79 & 90 & 81 & 40 \\
\hline Carbono ( $\left.\mathrm{g} \mathrm{kg}^{-1}\right)$ & 44 & 50 & 45 & 22 \\
\hline Relação C:N & $57: 1$ & $88: 1$ & $30: 1$ & $35: 1$ \\
\hline $\mathrm{pH}$ & 4,20 & 4,40 & 4,10 & 4,80 \\
\hline $\mathrm{CE}\left(\mathrm{mSm}^{-1}\right)$ & 1,19 & 1,76 & 1,08 & 4,26 \\
\hline Fósforo (mg kg-1) & 58,70 & 9,05 & 71,86 & 42,8 \\
\hline Potássio (mg kg-1) & 264,30 & 690,80 & 57,82 & 300,82 \\
\hline Magnésio (mg kg-1) & 107,30 & 23,50 & 105,3 & 454,30 \\
\hline Cálcio $\left(\mathrm{mg} \mathrm{kg}^{-1}\right)$ & 9,00 & 11,50 & 7,10 & 464,50 \\
\hline Enxofre $\left(\mathrm{mg} \mathrm{kg}^{-1}\right)$ & 8,10 & 25,95 & 3,09 & 62,78 \\
\hline Boro (mg kg-1) & 0,078 & 0,0 & 0,014 & 0,00 \\
\hline Ferro $\left(\mathrm{mg} \mathrm{kg}^{-1}\right)$ & 0,10 & 0,25 & 0,30 & 0,07 \\
\hline Manganês (mg kg-1) & 1,20 & 0,78 & 1,21 & 0,13 \\
\hline Cobre $\left(\mathrm{mg} \mathrm{kg}^{-1}\right)$ & 0,07 & 0,29 & 0,24 & 0,06 \\
\hline Zinco (mg kg-1) & 0,07 & 0,69 & 0,09 & 0,01 \\
\hline
\end{tabular}

(1) Uma parte de substrato para duas partes de água destilada.

macronutrientes no sistema radicular, na condição de mais e de menos água ( $6 \mathrm{~mm}$ diários), o que foi verificado pela alta mortalidade das mudas (Lopes et al., 2005), embora o substrato MIX tenha registrado maior acúmulo de $\mathrm{N}$ e semelhante de $\mathrm{P}, \mathrm{K}$ e S.

$\mathrm{O} \mathrm{P}$, o $\mathrm{S}$ e o $\mathrm{Mg}$ foram os nutrientes cujos acúmulos foram semelhantes nos substratos MIX, FB e CATV. Dentre os macronutrientes, $\mathrm{P}$ e $\mathrm{S}$ foram os menos acumulados nas raízes. Com relação ao $\mathrm{P}$, esse baixo acúmulo registrado pode estar associado à baixa mobilidade do nutriente nos substratos, à semelhança do que ocorre nos solos com umidade baixa (Lima et al., 1997). O fato de em CPV terem sido registrados acúmulos maiores pode estar relacionado a uma possível maior facilidade de absorção de água pelas mudas neste substrato e, dessa maneira, pelo maior acúmulo dos nutrientes. Os acúmulos neste substrato, quando submetido a $6 \mathrm{~mm} \mathrm{dia}^{-1}$, foram de $\mathrm{N}>\mathrm{K}>\mathrm{Mg}>\mathrm{Ca}>\mathrm{S}>\mathrm{P}$ e, quando submetido a $14 \mathrm{~mm} \mathrm{dia}^{-1}$, de $\mathrm{Ca}>\mathrm{N}>\mathrm{Mg}>\mathrm{K}>\mathrm{P}>\mathrm{S}$.

Essa tendência de maior acúmulo de macronutrientes de acordo com a disponibilidade de água também foi verificada por Lima et al. (1997) e Silva (2003). Na pesquisa de Lima et al. (1997), em mudas de eucalipto em vasos, foi observado que a absorção de todos os macronutrientes foi diminuída em função do estresse de água $(-0,05 \mathrm{MPa})$ em dois solos (argiloso e franco) e, na de Silva (2003), que utilizou mudas de $E$. grandis obtidas de sementes (em tubetes), na tensão de -0,01 $\mathrm{MPa}$, foram registrados acúmulos de nutrientes nas raízes, que, embora não associados ao substrato, seguiram a mesma tendência obtida nesta pesquisa.

Na parte aérea, assim como no sistema radicular, verifica-se tendência (Quadros 2 e 3) de acúmulo maior de $\mathrm{N}, \mathrm{P}, \mathrm{K}, \mathrm{Ca}, \mathrm{Mg}$ e S quanto maior a lâmina de irrigação a que as mudas foram submetidas, do mesmo modo que Lima et al. (1997) observaram nas folhas, em condições de maior disponibilidade de água, em dois tipos distintos de solo. No substrato CPV foi verificado o maior teor inicial de Ca (Quadro 1), o que refletiu em menor acúmulo de K na parte aérea, devido ao efeito antagônico entre esses nutrientes, conforme também constataram Trigueiro \& Guerrini (2003). Em condições tanto de menor disponibilidade de água $\left(6 \mathrm{~mm} \mathrm{dia}{ }^{-1}\right)$ como de maior $\left(14 \mathrm{~mm} \mathrm{dia}^{-1}\right)$, praticamente não houve diferença entre os acúmulos de macronutrientes na parte aérea, considerando os substratos.

De modo geral, independentemente dos substratos e da irrigação, os acúmulos foram de $\mathrm{K}>\mathrm{N}>\mathrm{Ca}>$ $\mathrm{Mg}>\mathrm{P}>\mathrm{S}$, sendo, à semelhança do que ocorreu nas raízes, o $\mathrm{P}$ e o $\mathrm{S}$ os menos absorvidos pelas mudas. $\mathrm{O}$ $\mathrm{Ca}$, que, embora em condições de baixa umidade, é também, como o $\mathrm{P}$, bastante afetado, não foi um dos nutrientes menos absorvidos. 
Quadro 2. Conteúdo de macronutrientes no sistema radicular e na parte aérea das mudas de Eucalyptus grandis em função das lâminas de irrigação e dos substratos

\begin{tabular}{|c|c|c|c|c|c|c|c|c|}
\hline \multirow{3}{*}{ Lâmina } & \multicolumn{4}{|c|}{ Sistema radicular } & \multicolumn{4}{|c|}{ Parte aéraa } \\
\hline & \multicolumn{8}{|c|}{ Substrato } \\
\hline & MIX & FB & CPV & CATV & MIX & FB & CPV & CATV \\
\hline $\mathrm{mm} \mathrm{dia-1}$ & \multicolumn{8}{|c|}{ N (mg/planta) } \\
\hline 6 & 3,33 & 3,98 & 4,07 & 2,12 & 8,58 & 10,15 & 9,38 & 5,49 \\
\hline 8 & 4,18 & 3,07 & 4,52 & 3,94 & 15,88 & 16,66 & 17,65 & 12,52 \\
\hline 10 & 5,21 & 4,59 & 4,29 & 4,39 & 23,06 & 20,26 & 19,10 & 19,02 \\
\hline 12 & 5,87 & 5,43 & 6,18 & 5,37 & 21,99 & 20,44 & 20,72 & 22,10 \\
\hline 14 & 7,02 & 5,24 & 4,89 & 5,57 & 27,32 & 24,01 & 24,12 & 24,43 \\
\hline Efeitos ${ }^{(1)}$ & $\mathrm{Qi}^{* *}$ & $\mathrm{Qi}^{* *}$ & $\mathrm{Qi}^{*}$ & $\mathrm{Qi}^{* *}$ & $\mathrm{Qi}^{* *}$ & $\mathrm{Qi}^{* *}$ & $\mathrm{Qi}^{* *}$ & $\mathrm{Qi}^{* *}$ \\
\hline \multirow[t]{2}{*}{ CV (\%) } & 12,16 & 18,30 & 16,60 & 13,00 & 14,30 & 18,00 & 19,50 & 13,70 \\
\hline & \multicolumn{8}{|c|}{ P (mg/planta) } \\
\hline 6 & 0,56 & 0,66 & 0,70 & 0,31 & 1,32 & 1,46 & 1,64 & 0,87 \\
\hline 8 & 0,58 & 0,52 & 1,39 & 0,60 & 2,24 & 2,35 & 3,42 & 2,11 \\
\hline 10 & 0,67 & 0,73 & 1,51 & 0,71 & 3,49 & 3,18 & 3,73 & 3,12 \\
\hline 12 & 0,98 & 0,88 & 1,90 & 0,86 & 3,51 & 3,01 & 4,75 & 3,95 \\
\hline 14 & 1,16 & 0,94 & 1,72 & 0,97 & 4,54 & 3,86 & 4,93 & 3,93 \\
\hline Efeitos $^{(1)}$ & $\mathrm{Qi}^{* *}$ & $\mathrm{Qi}^{* *}$ & $\mathrm{Qi}^{* *}$ & $\mathrm{Qi}^{* *}$ & $\mathrm{Qi}^{* *}$ & $\mathrm{Qi}^{* *}$ & $\mathrm{Qi}^{* *}$ & $\mathrm{Qi}^{* *}$ \\
\hline \multirow[t]{2}{*}{ CV (\%) } & 15,50 & 17,40 & 11,00 & 14,00 & 14,20 & 14,10 & 12,20 & 13,30 \\
\hline & \multicolumn{8}{|c|}{$\mathrm{K}$ (mg/planta) } \\
\hline 6 & 1,66 & 2,18 & 2,09 & 0,93 & 8,85 & 11,60 & 10,94 & 6,10 \\
\hline 8 & 1,38 & 1,71 & 2,63 & 1,70 & 14,66 & 16,75 & 18,2 & 12,67 \\
\hline 10 & 1,81 & 2,28 & 2,25 & 1,67 & 22,99 & 22,24 & 20,59 & 17,17 \\
\hline 12 & 2,20 & 2,31 & 3,09 & 1,84 & 23,53 & 23,66 & 25,82 & 22,56 \\
\hline 14 & 2,91 & 2,53 & 3,12 & 2,21 & 28,14 & 28,91 & 27,80 & 25,44 \\
\hline Efeitos $^{(1)}$ & $\mathrm{Qi}^{* *}$ & $\mathrm{Qi}^{*}$ & $Q \mathrm{i}^{* *}$ & $\mathrm{Qi}^{* *}$ & $\mathrm{Qi}^{* *}$ & $\mathrm{Qi}^{* *}$ & $\mathrm{Qi}^{* *}$ & $\mathrm{Qi}^{* *}$ \\
\hline \multirow[t]{2}{*}{ CV (\%) } & 8,11 & 12,48 & 14,80 & 15,20 & 12,30 & 12,60 & 11,50 & 9,50 \\
\hline & \multicolumn{8}{|c|}{$\mathrm{Ca}$ (mg/planta) } \\
\hline 6 & 1,22 & 1,27 & 1,71 & 0,57 & 3,08 & 4,06 & 4,69 & 2,02 \\
\hline 8 & 1,73 & 2,49 & 5,08 & 1,62 & 5,32 & 7,11 & 9,63 & 5,49 \\
\hline 10 & 1,93 & 3,24 & 5,15 & 1,74 & 8,89 & 10,94 & 9,79 & 6,08 \\
\hline 12 & 2,46 & 3,84 & 7,29 & 2,16 & 8,97 & 12,09 & 13,59 & 8,36 \\
\hline 14 & 2,45 & 3,86 & 5,82 & 3,02 & 12,03 & 16,16 & 15,04 & 11,65 \\
\hline Efeitos $^{(1)}$ & $\mathrm{Qi}^{* *}$ & $\mathrm{Qi}^{* *}$ & $\mathrm{Qi}^{* *}$ & $\mathrm{Qi}^{* *}$ & $\mathrm{Qi}^{* *}$ & $\mathrm{Qi}^{* *}$ & $\mathrm{Qi}^{* *}$ & $\mathrm{Qi}^{* *}$ \\
\hline \multirow[t]{2}{*}{ CV (\%) } & 14,61 & 12,39 & 15,80 & 25,70 & 15,00 & 9,20 & 12,50 & 18,00 \\
\hline & \multicolumn{8}{|c|}{$\mathrm{Mg}$ (mg/planta) } \\
\hline 6 & 1,19 & 1,18 & 1,95 & 0,61 & 1,52 & 1,88 & 1,91 & 0,96 \\
\hline 8 & 1,24 & 0,99 & 4,25 & 1,24 & 2,34 & 2,97 & 3,40 & 2,09 \\
\hline 10 & 1,44 & 1,55 & 3,31 & 1,27 & 3,95 & 4,34 & 3,64 & 2,77 \\
\hline 12 & 1,79 & 1,95 & 3,66 & 1,70 & 3,76 & 4,58 & 4,77 & 3,83 \\
\hline 14 & 2,03 & 1,87 & 4,67 & 2,09 & 5,26 & 5,98 & 5,15 & 4,84 \\
\hline Efeitos $^{(1)}$ & $\mathrm{Qi}^{* *}$ & $\mathrm{Qi}^{* *}$ & $\mathrm{Qi}^{* *}$ & $\mathrm{Qi}^{* *}$ & $\mathrm{Qi}^{* *}$ & $\mathrm{Qi}^{* *}$ & $\mathrm{Qi}^{* *}$ & $\mathrm{Qi}^{* *}$ \\
\hline \multirow[t]{2}{*}{ CV (\%) } & 13,17 & 16,14 & 23,32 & 18,70 & 16,80 & 10,40 & 14,10 & 12,10 \\
\hline & \multicolumn{8}{|c|}{$\mathrm{S}$ (mg/planta) } \\
\hline 6 & 0,60 & 0,73 & 0,79 & 0,40 & 0,73 & 1,01 & 0,93 & 0,53 \\
\hline 8 & 0,80 & 0,70 & 1,24 & 0,71 & 1,43 & 1,77 & 2,06 & 1,32 \\
\hline 10 & 0,94 & 0,99 & 1,34 & 0,80 & 2,37 & 2,33 & 2,36 & 1,77 \\
\hline 12 & 1,19 & 1,11 & 1,76 & 1,05 & 2,10 & 2,42 & 2,64 & 2,14 \\
\hline 14 & 1,40 & 1,21 & 1,59 & 1,23 & 2,47 & 2,87 & 2,84 & 2,70 \\
\hline Efeitos $^{(1)}$ & $\mathrm{Qi}^{* *}$ & $\mathrm{Qi}^{* *}$ & $\mathrm{Qi}^{* *}$ & $\mathrm{Qi}^{* *}$ & $\mathrm{Qi}^{* *}$ & $\mathrm{Qi}^{* *}$ & $\mathrm{Qi}^{* *}$ & $\mathrm{Qi}^{* *}$ \\
\hline CV (\%) & 15,90 & 15,56 & 11,34 & 16,20 & 15,50 & 15,10 & 17,40 & 13,50 \\
\hline
\end{tabular}

${ }^{(1)}$ Li- efeito linear; Qi- efeito quadrático; NS: não-significativo. * $: \mathrm{P}<0,05 ; * *$ : $\mathrm{P}<0,01$. 
Quadro 3. Equações de regressão para conteúdo de macronutrientes no sistema radicular e na parte aérea de mudas de Eucalyptus grandis em função das lâminas de irrigação e dos substratos

\begin{tabular}{|c|c|c|c|}
\hline \multirow[t]{2}{*}{ Substrato } & Sistema radicular & \multicolumn{2}{|l|}{ Parte aérea } \\
\hline & \multicolumn{3}{|c|}{ Nitrogênio } \\
\hline MIX & $y=0,9595+0,3724 \cdot x+0,0041 \cdot x^{2}$ & $r^{2}=0,83^{* *} \quad y=-22,4806+6,5382 \cdot x-0,2179 \cdot x^{2}$ & $\mathrm{r}^{2}=0,86^{* *}$ \\
\hline FB & $y=3,300786-0,0348 \cdot x+0,0140 \cdot x^{2}$ & ${ }^{2} r^{2}=0,46^{* *} \quad y=-12,7474+4,9010 \cdot x-0,1663 \cdot x^{2}$ & $\mathrm{r}^{2}=0,69^{* *}$ \\
\hline $\mathrm{CPV}$ & $\mathrm{y}=0,9011+0,6525 \cdot \mathrm{x}-0,0244 \cdot \mathrm{x}^{2}$ & $r^{2}=0,31^{*} \quad y=-13,7879+5,0413 \cdot x-0,1707 \cdot x^{2}$ & $\mathrm{r}^{2}=0,68^{* *}$ \\
\hline \multirow[t]{2}{*}{ CATV } & $y=-4,338+1,3849 \cdot x-0,0484 \cdot x^{2}$ & $\mathrm{r}^{2}=0,85^{* *} \mathrm{y}=-28,0856+6,9529 \cdot \mathrm{x}-0,2290 \cdot \mathrm{x}^{2}$ & $\mathrm{r}^{2}=0,91 * *$ \\
\hline & \multicolumn{3}{|c|}{ Fósforo } \\
\hline MIX & $y=0,8633-0,1111 \cdot x+0,0096 \cdot x^{2}$ & $r^{2}=0,80^{* *} \quad y=-2,5213+0,7523 \cdot x-0,0183 \cdot x^{2}$ & $\mathrm{r}^{2}=0,88^{* *}$ \\
\hline $\mathrm{FB}$ & $y=0,8684-0,08052 \cdot x+0,0063 \cdot x^{2}$ & $r^{2}=0,56^{* *} \quad y=-1,7251+0,6585 \cdot x-0,0193 \cdot x^{2}$ & $\mathrm{r}^{2}=0,82^{* *}$ \\
\hline $\mathrm{CPV}$ & $y=-2,2322+0,6487 \cdot x-0,0260 \cdot x^{2}$ & $r^{2}=0,88^{* *} \quad y=-3,9929+1,2092 \cdot x-0,0411 \cdot x^{2}$ & $\mathrm{r}^{2}=0,88^{* *}$ \\
\hline \multirow[t]{2}{*}{ CATV } & $y=-0,6184+0,1920 \cdot x-0,0057 \cdot x^{2}$ & $r^{2}=0,87^{* *} \quad y=-5,6076+1,3597 \cdot x-0,0481 \cdot x^{2}$ & $\mathrm{r}^{2}=0,92^{* *}$ \\
\hline & \multicolumn{3}{|c|}{ Potássio } \\
\hline MIX & $y=3,5123-0,52583 \cdot x+0,0346 \cdot x^{2}$ & $r^{2}=0,93^{* *} \quad y=-20,8103+6,0075 \cdot x-0,1818 \cdot x^{2}$ & $\mathrm{r}^{2}=0,90^{* *}$ \\
\hline FB & $\mathrm{y}=2,9324-0,2355 \cdot \mathrm{x}+0,0150 \cdot \mathrm{x}^{2}$ & $r^{2}=0,41^{*} \quad y=-6,4661+3,4535 \cdot x-0,0689 \cdot x^{2}$ & $\mathrm{r}^{2}=0,86^{* *}$ \\
\hline $\mathrm{CPV}$ & $y=1,7037+0,0551 \cdot x+0,0035 \cdot x^{2}$ & $r^{2}=0,50^{* *} \quad y=-12,6821+4,8231 \cdot x-0,1378 \cdot x^{2}$ & $\mathrm{r}^{2}=0,88^{* *}$ \\
\hline \multirow[t]{2}{*}{ CATV } & $\mathrm{y}=-0,6534+0,3454 \cdot \mathrm{x}-0,0105 \cdot \mathrm{x}^{2}$ & $r^{2}=0,74^{* *} \quad y=-18,2041+4,7561 \cdot x-0,1164 \cdot x^{2}$ & $\mathrm{r}^{2}=0,96^{* *}$ \\
\hline & \multicolumn{3}{|c|}{ Cálcio } \\
\hline MIX & $\mathrm{y}=-0,7864+0,4098 \cdot \mathrm{x}-0,0125 \cdot \mathrm{x}^{2}$ & $\mathrm{r}^{2}=0,75^{* *} \quad \mathrm{y}=-6,1581+1,7372 \cdot \mathrm{x}-0,0329 \cdot \mathrm{x}^{2}$ & $\mathrm{r}^{2}=0,89 * *$ \\
\hline $\mathrm{FB}$ & $\mathrm{y}=-4,5121+1,2375 \cdot \mathrm{x}-0,0456 \cdot \mathrm{x}^{2}$ & $\mathrm{r}^{2}=0,89 * * \quad y=-5,5517+1,6848 \cdot \mathrm{x}-0,0114 \cdot \mathrm{x}^{2}$ & $\mathrm{r}^{2}=0,96^{* *}$ \\
\hline $\mathrm{CPV}$ & $\mathrm{y}=-12,7158+3,2396 \cdot \mathrm{x}-0,1358 \cdot \mathrm{x}^{2}$ & $\mathrm{r}^{2}=0,85^{* *} \quad \mathrm{y}=-7,2822+2,4284 \cdot \mathrm{x}-0,0598 \cdot \mathrm{x}^{2}$ & $\mathrm{r}^{2}=0,89 * *$ \\
\hline \multirow[t]{2}{*}{ CATV } & $y=-1,0368+0,3013 \cdot x-0,0015 \cdot x^{2}$ & $\mathrm{r}^{2}=0,76^{* *} \mathrm{y}=-2,1422+0,6272 \cdot \mathrm{x}+0,0240 \cdot \mathrm{x}^{2}$ & $\mathrm{r}^{2}=0,89^{* *}$ \\
\hline & \multicolumn{3}{|c|}{ Magnésio } \\
\hline MIX & $\mathrm{y}=1,2939-0,0773 \cdot \mathrm{x}+0,0094 \cdot \mathrm{x}^{2}$ & $\mathrm{r}^{2}=0,75^{* *} \quad \mathrm{y}=-1,8027+0,6022 \cdot \mathrm{x}-0,0079 \cdot \mathrm{x}^{2}$ & $\mathrm{r}^{2}=0,85^{* *}$ \\
\hline FB & $\mathrm{y}=0,4352+0,0961 \cdot \mathrm{x}+0,0010 \cdot \mathrm{x}^{2}$ & $\mathrm{r}^{2}=0,68^{* *} \quad \mathrm{y}=-1,7851+0,6706 \cdot \mathrm{x}-0,0090 \cdot \mathrm{x}^{2}$ & $\mathrm{r}^{2}=0,94 * *$ \\
\hline $\mathrm{CPV}$ & $y=-4,8957+1,525 \cdot x-0,0591 \cdot x^{2}$ & $r^{2}=0,60^{* *} \quad y=-2,3487+0,8695 \cdot x-0,0238 \cdot x^{2}$ & $\mathrm{r}^{2}=0,84 * *$ \\
\hline \multirow[t]{2}{*}{ CATV } & $\mathrm{y}=-0,4533+0,1993 \cdot \mathrm{x}-0,0015 \cdot \mathrm{x}^{2}$ & $r^{2}=0,80^{* *} \quad y=-1,6483+0,42983 \cdot x+0,0023 \cdot x^{2}$ & $\mathrm{r}^{2}=0,95^{* *}$ \\
\hline & \multicolumn{3}{|c|}{ Enxofre } \\
\hline MIX & $y=0,1996+0,0533 \cdot x+0,0023 \cdot x^{2}$ & $\mathrm{r}^{2}=0,79 * * \quad \mathrm{y}=-3,2961+0,8687 \cdot \mathrm{x}-0,0331 \cdot \mathrm{x}^{2}$ & $\mathrm{r}^{2}=0,85^{* *}$ \\
\hline FB & $\mathrm{y}=0,4096+0,0374 \cdot \mathrm{x}+0,0015 \cdot \mathrm{x}^{2}$ & $\mathrm{r}^{2}=0,67^{* *} \quad \mathrm{y}=-1,8858+0,6047 \cdot \mathrm{x}-0,0193 \cdot \mathrm{x}^{2}$ & $\mathrm{r}^{2}=0,83^{* *}$ \\
\hline $\mathrm{CPV}$ & $\mathrm{y}=-1,2729+0,4443 \cdot \mathrm{x}-0,0169 \cdot \mathrm{x}^{2}$ & $\mathrm{r}^{2}=0,84^{* *} \quad \mathrm{y}=-3,1031+0,8876 \cdot \mathrm{x}-0,0334 \cdot \mathrm{x}^{2}$ & $\mathrm{r}^{2}=0,78^{* *}$ \\
\hline CATV & $y=-0,311+0,1316 \cdot x-0,0016 \ldots x^{2}$ & $r^{2}=0,84^{* *} \quad y=-1,7602+0,4473 \cdot x-0,0095 \cdot x^{2}$ & $\mathrm{r}^{2}=0,92^{* *}$ \\
\hline
\end{tabular}

$*: \mathrm{P}<0,05 ; * *: \mathrm{P}<0,01$.

As recomendações nutricionais para a parte aérea das mudas são em grande parte relacionadas aos teores dos nutrientes nas folhas (Gonçalves \& Poggiani, 1996; Higashi et al., 2000; Silveira et al., 2001) e, do mesmo modo como para os acúmulos de nutrientes no sistema radicular, não envolvem principalmente as questões hídricas, que conseqüentemente reportariam a acúmulos distintos e específicos para cada manejo hídrico. Justamente devido a isso e aos diferentes substratos usados nas pesquisas mencionadas, é possível que os resultados registrados nesta pesquisa, para acúmulos de nutrientes na parte aérea, estejam adequados para o estabelecimento de critérios nutricionais de qualidade.

\section{Concentração de micronutrientes no sistema radicular e na parte aérea das mudas}

Considerando o acúmulo de micronutrientes no sistema radicular (Quadros 4 e 5), observam-se comportamentos dos substratos e dos micronutrientes distintos de acordo com as lâminas de irrigação aplicadas. Para o substrato MIX, as lâminas de irrigação tiveram pouca ou nenhuma influência no 
acúmulo de micronutrientes nas raízes. Quanto ao substrato $\mathrm{FB}$, os registros de micronutrientes nas raízes foram tanto maiores quanto maior $o$ fornecimento de água.

FB e CPV (embora não-significativos para o boro em CPV, conforme Quadros 4 e 5) podem ser considerados como os substratos que possibilitaram às mu- das registrarem os maiores acúmulos de micronutrientes em condições tanto de mais água $(14 \mathrm{~mm})$ como de menos (6 mm). No caso do substrato FB, os maiores acúmulos registrados foram do $\mathrm{Zn}>\mathrm{B}>\mathrm{Cu}$ e, do substrato $\mathrm{CPV}$, do $\mathrm{Fe}>\mathrm{Mn}>\mathrm{B}$.

Também no caso dos micronutrientes, a literatura não estabelece uma faixa adequada de nutrientes que

Quadro 4. Conteúdo de micronutrientes no sistema radicular e na parte aérea das mudas de Eucalyptus grandis de acordo com as lâminas de irrigação e os substratos

\begin{tabular}{|c|c|c|c|c|c|c|c|c|}
\hline \multirow{3}{*}{ Lâmina } & \multicolumn{4}{|c|}{ Sistema radicular } & \multicolumn{4}{|c|}{ Parte aérea } \\
\hline & \multicolumn{8}{|c|}{ Substrato } \\
\hline & MIX & FB & CPV & CATV & MIX & FB & $\mathrm{CPV}$ & CATV \\
\hline $\mathrm{mm} \mathrm{dia}^{-1}$ & \multicolumn{8}{|c|}{$\mathrm{B}(\mu \mathrm{g} / \mathrm{planta})$} \\
\hline 6 & 12,26 & 14,23 & 14,21 & 6,31 & 12,43 & 15,43 & 16,16 & 6,66 \\
\hline 8 & 17,59 & 18,86 & 22,42 & 17,15 & 18,96 & 30,15 & 29,54 & 17,59 \\
\hline 10 & 17,98 & 18,18 & 15,88 & 12,31 & 33,41 & 33,86 & 31,30 & 21,67 \\
\hline 12 & 19,36 & 22,86 & 20,85 & 13,29 & 40,14 & 48,48 & 56,23 & 40,79 \\
\hline 14 & 18,64 & 26,64 & 18,43 & 24,77 & 39,40 & 50,10 & 48,61 & 38,02 \\
\hline Efeitos $^{(1)}$ & $\mathrm{Qi} * *$ & $\mathrm{Qi} * *$ & NS & $\mathrm{Qi}^{* *}$ & $\mathrm{Qi}^{* *}$ & $\mathrm{Qi}^{* *}$ & $\mathrm{Qi}^{* *}$ & $\mathrm{Q}^{* *}$ \\
\hline CV (\%) & 12,80 & 15,40 & 19,00 & 30,20 & 17,20 & 15,00 & 20,00 & 20,50 \\
\hline \multicolumn{9}{|c|}{$\mathrm{Cu}(\mu \mathrm{g} / \mathrm{planta})$} \\
\hline 6 & 1,02 & 2,86 & 1,33 & 0,41 & 1,07 & 1,33 & 1,08 & 0,67 \\
\hline 8 & 3,42 & 12,83 & 7,82 & 3,82 & 0,94 & 4,87 & 2,34 & 2,26 \\
\hline 10 & 3,63 & 16,26 & 6,41 & 1,49 & 3,30 & 7,34 & 2,11 & 1,02 \\
\hline 12 & 5,11 & 23,71 & 8,93 & 2,21 & 2,14 & 9,08 & 2,79 & 1,14 \\
\hline 14 & 5,99 & 24,95 & 8,19 & 12,48 & 3,33 & 9,12 & 3,51 & 3,96 \\
\hline Efeitos $^{(1)}$ & $\mathrm{Qi}^{* *}$ & $\mathrm{Qi}^{* *}$ & $\mathrm{Qi}^{* *}$ & $\mathrm{Qi}^{*}$ & $\mathrm{Qi}^{*}$ & $\mathrm{Qi}^{* *}$ & $\mathrm{Qi}^{* *}$ & NS \\
\hline $\mathrm{CV}(\%)$ & 49,20 & 24,90 & 28,80 & 126,50 & 52,30 & 16,90 & 27,30 & 100,18 \\
\hline \multicolumn{9}{|c|}{$\mathrm{Fe}(\mu \mathrm{g} / \mathrm{planta})$} \\
\hline 6 & 547,20 & 442,34 & 900,73 & 274,72 & 82,76 & 104,43 & 81,46 & 47,64 \\
\hline 8 & 790,8 & 388,5 & 2098,3 & 533,3 & 73,49 & 100,82 & 119,77 & 82,6 \\
\hline 10 & 653,3 & 620,9 & 2267,7 & 600,1 & 144,87 & 164,69 & 136,09 & 91,49 \\
\hline 12 & 946,1 & 989,4 & 3742,0 & 915,2 & 138,66 & 194,10 & 162,96 & 145,56 \\
\hline 14 & 985,0 & 993,7 & 3107,2 & 1331,5 & 164,64 & 157,03 & 163,96 & 173,86 \\
\hline Efeitos $^{(1)}$ & $\mathrm{Qi} *$ & $\mathrm{Qi} * *$ & $\mathrm{Qi}^{* *}$ & $\mathrm{Qi}^{* *}$ & $\mathrm{Qi} * *$ & $\mathrm{Qi}^{* *}$ & $\mathrm{Qi}^{* *}$ & $\mathrm{Qi} * *$ \\
\hline CV (\%) & 28,30 & 25,80 & 19,50 & 27,30 & 19,80 & 22,90 & 13,10 & 16,80 \\
\hline \multicolumn{9}{|c|}{ Mn ( $\mu \mathrm{g} /$ planta $)$} \\
\hline 6 & 28,65 & 26,22 & 31,84 & 15,58 & 209,83 & 182,65 & 186,78 & 136,28 \\
\hline 8 & 41,27 & 18,77 & 71,75 & 34,09 & 390,27 & 195,15 & 260,43 & 343,79 \\
\hline 10 & 40,26 & 21,38 & 62,38 & 36,36 & 510,90 & 208,22 & 262,97 & 435,19 \\
\hline 12 & 52,90 & 30,39 & 85,14 & 46,63 & 455,29 & 205,86 & 362,22 & 602,38 \\
\hline 14 & 49,38 & 33,14 & 80,86 & 42,89 & 649,22 & 267,44 & 500,49 & 558,82 \\
\hline Efeitos $^{(1)}$ & $\mathrm{Qi}^{* *}$ & $\mathrm{Qi}^{*}$ & $\mathrm{Qi}^{* *}$ & $\mathrm{Qi}^{* *}$ & $\mathrm{Qi}^{* *}$ & $\mathrm{Qi}^{*}$ & $\mathrm{Qi}^{* *}$ & $\mathrm{Qi}^{* *}$ \\
\hline CV (\%) & 14,03 & 26,80 & 20,70 & 22,70 & 16,00 & 15,50 & 15,20 & 21,20 \\
\hline \multicolumn{9}{|c|}{$\mathrm{Zn}(\mu \mathrm{g} /$ planta $)$} \\
\hline 6 & 10,71 & 15,91 & 11,82 & 5,89 & 14,66 & 21,45 & 14,68 & 8,25 \\
\hline 8 & 13,23 & 29,44 & 17,27 & 18,68 & 27,22 & 36,32 & 26,42 & 22,17 \\
\hline 10 & 17,60 & 44,78 & 18,81 & 12,57 & 33,02 & 50,62 & 26,96 & 25,04 \\
\hline 12 & 21,40 & 56,58 & 21,68 & 15,75 & 25,28 & 36,12 & 26,51 & 22,43 \\
\hline 14 & 23,43 & 60,38 & 22,58 & 42,50 & 25,00 & 42,37 & 24,61 & 28,91 \\
\hline Efeitos $^{(1)}$ & $\mathrm{Qi}^{* *}$ & $\mathrm{Qi}^{* *}$ & $\mathrm{Qi}^{* *}$ & $\mathrm{Qi}^{*}$ & $\mathrm{Qi}^{* *}$ & $\mathrm{Qi}^{* *}$ & $\mathrm{Qi}^{* *}$ & $\mathrm{Qi}^{* *}$ \\
\hline CV (\%) & 17,40 & 22,40 & 11,50 & 82,60 & 21,00 & 19,30 & 15,10 & 26,50 \\
\hline
\end{tabular}

(1) $\mathrm{Li}$ - efeito linear; $\mathrm{Qi}$ - efeito quadrático; NS: não-significativo; * $: \mathrm{P}<0,05 ;{ }^{* *}: \mathrm{P}<0,01$. 
Quadro 5. Equações de regressão para conteúdo de micronutrientes no sistema radicular e na parte aérea de mudas de Eucalyptus grandis em função das lâminas de irrigação e dos substratos

$\begin{array}{lll}\text { Substrato } & \text { Sistema radicular } & \text { Parte aérea }\end{array}$

\begin{tabular}{|c|c|c|c|c|}
\hline & \multicolumn{4}{|c|}{ Boro } \\
\hline MIX & $y=-8,3436+4,6923 \cdot x-0,1983 \cdot x^{2}$ & $r^{2}=0,59 * *$ & $y=-45,2891+11,7116 \cdot x-0,3978 \cdot x^{2} r^{2}$ & $r^{2}=0,85^{* *}$ \\
\hline FB & $y=11,7709+0,1313 \cdot x+0,0655 \cdot x^{2}$ & $r^{2}=0,67 * *$ & $y=-33,3836+9,8514 \cdot x-0,2734 \cdot x^{2}$ & $r^{2}=0,87 * *$ \\
\hline $\mathrm{CPV}$ & NS & & $y=-40,3362+11,2996 \cdot x-0,3360 \cdot x^{2} \quad r$ & $r^{2}=0,79^{* *}$ \\
\hline \multirow[t]{2}{*}{ CATV } & $y=9,8706-0,8769 \cdot x+0,1265 \cdot x^{2}$ & $\mathrm{r}^{2}=0,57 * *$ & $y=-38,3434+8,7154 \cdot x-0,2210 \cdot x^{2}$ & $\mathrm{r}^{2}=0,87 * *$ \\
\hline & \multicolumn{4}{|c|}{ Cobre } \\
\hline MIX & $y=-4,8940+1,2136 \cdot x-0,0316 \cdot x^{2}$ & $r^{2}=0,48^{* *}$ & $y=-2,1516+0,6010 \cdot x-0,0158 \cdot x^{2}$ & $r^{2}=0,38 * *$ \\
\hline FB & $y=-33,4844+7,5509 \cdot x-0,2399 \cdot x^{2}$ & $r^{2}=0,82^{* *}$ & $y=-16,2295+3,7461 \cdot x-0,1378 \cdot x^{2}$ & $r^{2}=0,90 * *$ \\
\hline $\mathrm{CPV}$ & $y=-18,1468+4,4956 \cdot x-0,18776 \cdot x^{2}$ & $\mathrm{r}^{2}=0,67 * *$ & $y=-0,5489+0,3224 \cdot x-0,0029 \cdot x^{2}$ & $\mathrm{r}^{2}=0,61 * *$ \\
\hline \multirow[t]{2}{*}{ CATV } & $y=-20,3561+4,8602 \cdot x-0,2993 \cdot x^{2}$ & $\mathrm{r}^{2}=0,38^{*}$ & NS & \\
\hline & \multicolumn{4}{|c|}{ Ferro } \\
\hline MIX & $\mathrm{y}=358,8119+31,2450 \cdot \mathrm{x}+1,0150 \cdot \mathrm{x}^{2}$ & $\mathrm{r}^{2}=0,34^{*}$ & $y=-5,2012+13,9730 \cdot x-0,1263 \cdot x^{2}$ & $\mathrm{r}^{2}=0,68^{* *}$ \\
\hline FB & $y=249,9861-5,0064 \cdot x+4,5096 \cdot x^{2}$ & $r^{2}=0,69 * *$ & $y=-121,5882+46,1324 \cdot x-1,8104 \cdot x^{2} r$ & $\mathrm{r}^{2}=0,50 * *$ \\
\hline $\mathrm{CPV}$ & $y=-4482,0412+1145,6433 \cdot x-42,1407 x^{2}$ & $r^{2}=0,81 * *$ & $y=-76,5120+33,2916 \cdot x-1,144 \cdot x^{2}$ & $r^{2}=0,78^{* *}$ \\
\hline \multirow[t]{2}{*}{ CATV } & $y=409,352-76,5586 \cdot x+10,0666 \cdot x^{2}$ & $r^{2}=0,79^{* *}$ & $y=2,8506+4,3958 \cdot x+0,5687 \cdot x^{2}$ & $\mathrm{r}^{2}=0,88^{* *}$ \\
\hline & \multicolumn{4}{|c|}{ Manganês } \\
\hline MIX & $y=-14,6581+9,3076 \cdot x-0,3326 \cdot x^{2}$ & $\mathrm{r}^{2}=0,67 * *$ & $y=-274,0299+100,5004 \cdot x-2,6655 \cdot x^{2}$ & $\mathrm{r}^{2}=0,81^{* *}$ \\
\hline FB & $y=57,3026-8,3043 \cdot x+0,4789 \cdot x^{2}$ & $\mathrm{r}^{2}=0,36^{*}$ & $y=257,6436-20,5345 \cdot x+1,4774 \cdot x^{2}$ & $\mathrm{r}^{2}=0,45^{* *}$ \\
\hline CPV & $y=-81,7540+25,6654 \cdot x-1,0047 \cdot x^{2}$ & $r^{2}=0,65^{* *}$ & $y=321,1494-44,2306 \cdot x+4,0346 \cdot x^{2}$ & $\mathrm{r}^{2}=0,85^{* *}$ \\
\hline \multirow[t]{2}{*}{ CATV } & $y=-58,4314+16,3927 \cdot x-0,6517 \cdot x^{2}$ & $\mathrm{r}^{2}=0,67 * *$ & $y=-836,9844+207,4531 \cdot x-7,6135 \cdot x^{2}$ & $r^{2}=0,80^{* *}$ \\
\hline & \multicolumn{4}{|c|}{ Zinco } \\
\hline MIX & $y=-2,0905+2,2366 \cdot x-0,0278 \cdot x^{2}$ & $r^{2}=0,75^{* *}$ & $y=-48,875+14,9702 \cdot x-0,7019 \cdot x^{2}$ & $\mathrm{r}^{2}=0,55^{* *}$ \\
\hline FB & $y=-54,3821+14,0124 \cdot x-0,4104 \cdot x^{2}$ & $\mathrm{r}^{2}=0,79^{* *}$ & $y=-59,1041+18,5292 \cdot x-0,8223 \cdot x^{2}$ & $\mathrm{r}^{2}=0,60 * *$ \\
\hline CPV & $y=-7,2681+4,0646 \cdot x-0,13839 \cdot x^{2}$ & $r^{2}=0,79 * *$ & $y=-32,5985+11,0972 \cdot x-0,505 \cdot x^{2}$ & $\mathrm{r}^{2}=0,64^{* *}$ \\
\hline CATV & $y=45,0719-9,7766 \cdot x+0,6646 \cdot x^{2}$ & $r^{2}=0,36^{*}$ & $y=-32,8934+9,3543 \cdot x-0,3638 \cdot x^{2}$ & $\mathrm{r}^{2}=0,60^{* *}$ \\
\hline
\end{tabular}

NS: não-significativo; ${ }^{*}: \mathrm{P}<0,05 ;{ }^{* *}: \mathrm{P}<0,01$.

devam estar acumulados no sistema radicular, os quais qualificam as mudas nutricionalmente; embora as pesquisas abordem esses aspectos, não envolvem a questão da água e, ou, dos substratos. Nesse sentido, Silveira et al. (2001) apresentam faixas de acúmulo próximas às encontradas nesta pesquisa, para o $\mathrm{B}$ e o $\mathrm{Cu}$ (exceto para o substrato FB, cujos acúmulos obtidos foram superiores), e inferiores para o Mn e o $\mathrm{Zn}$; para o Fe, os acúmulos foram superiores em todos os substratos (exceto para o CPV). Já a pesquisa de Silva (2003) relaciona os acúmulos dos micronutrientes a duas tensões de retenção de água no substrato; nesse caso, os acúmulos foram semelhantes aos desta pesquisa, exceto para o $\mathrm{Cu}$, nas mudas produzidas no substrato $\mathrm{FB}$, e para o $\mathrm{Fe}$, nas mudas produzidas no substrato CPV.

O manejo hídrico influenciou o acúmulo dos micronutrientes na parte aérea em diferentes intensidades, conforme o substrato usado (Quadros 4 e 5). O acúmulo de $\mathrm{Zn}$ teve pequena variação, mostrando diferença entre as lâminas de irrigação somente para as mudas produzidas no substrato FB, justificado pelo maior teor inicial deste elemento antes da produção das mudas (Quadro 1). Embora os substratos CPV e CATV tenham registrado teores iniciais de micronutrientes muito distintos, tiveram comportamento semelhante, em função das lâminas de irrigação; o acúmulo do $\mathrm{B}$, do $\mathrm{Fe}$ e do $\mathrm{Mn}$ acompanhou o aumento da lâmina de irrigação, e o $\mathrm{Zn}$ e o $\mathrm{Cu}$ tiveram pouca variação, em razão desse aumento.

A preocupação com o acúmulo dos micronutrientes na parte aérea das plantas está mais relacionada aos sintomas de toxicidade, principalmente aqueles relacionados ao $\mathrm{B}$ e ao $\mathrm{Fe}$. As recomendações nutricionais aplicam-se mais às mudas clonais, principalmente às cepas dos minijardins. No entanto, substratos compostos por fibras de coco podem conter altas concentrações de B (não observado nesta pesquisa); no caso do Fe (normalmente em altas concentrações em substratos à base de cascas de pinus), foram reportados por Silveira (2001) acúmulos maiores (de 412 a $477 \mu \mathrm{g}$ planta $^{-1}$ de Fe) nas folhas do que os obtidos nesta pesquisa em todos os substratos, 
embora esses acúmulos não tenham sido tóxicos às plantas. Silva (2003) registrou acúmulos que, assim como nesta pesquisa, variaram de acordo com o manejo hídrico aplicado, sendo semelhantes apenas para o Mn e o Zn e notadamente inferiores aos demais.

\section{CONCLUSÕES}

1. Houve influência das lâminas de irrigação e dos substratos no acúmulo dos nutrientes no sistema radicular e na parte aérea das plantas de E. grandis.

2. Os substratos CPV e FB e as maiores lâminas brutas de irrigação diária $(10,12$ e $14 \mathrm{~mm})$ registraram os maiores acúmulos de nutrientes. CPV foi o melhor para o acúmulo dos macronutrientes nas raízes e também, juntamente com $\mathrm{FB}$, o melhor para o acúmulo dos micronutrientes nas raízes e na parte aérea. Não houve influência dos substratos no acúmulo dos macronutrientes na parte aérea, porém houve das lâminas de irrigação.

\section{LITERATURA CITADA}

ABAD, M.; MARTINEZ-HERRERO, M.D.; MARTINEZGARCIA, P.F. \& MARTINEZ-CORTS, J. Evaluación agronômica de los sustratos de cultivo. Jornadas de Sustratos. I. Actas Hortic., 11:141-154, 1992.

ABREU, M.F. Legislação de substrato para plantas. In: ENCONTRO BRASILEIRO SOBRE SUBSTRATOS PARA PLANTAS, 5., 2006, Ilhéus. Anais. Ilhéus, CEPLAC/ CEPEC. 2006. p.75-81.

ALVARENGA, R.C.; BARROS, N.F.; DANTAS, C.E.S. \& LOBÃO, D.E.V.P. Efeitos do conteúdo de água no solo e da poda de raízes sobre o crescimento de mudas de eucalipto. R. Árvore, 18:107-114, 1994.

BARROS, N.F.; NEVES, J.C.L. \& NOVAIS, R.F. Nutrição e adubação de eucalipto. Inf.Agropec., 18:70-75, 1997.

BRASIL. Ministério da Agricultura. Análise de corretivos, fertilizantes e inoculantes. Métodos Oficiais. Brasília, 1988. 110p.

BRASIL. Ministério da Agricultura. Pecuária e Abastecimento. Decreto Lei $\mathrm{n}^{\circ}$ 4954, de 14 de janeiro de 2004 - Instrução Normativa $\mathrm{n}^{\circ} 14$, de 15 de dezembro de 2004. Disponível em: http://www.pr.gov.br/seab /in_14_04_anexo. pdf\#search $=\% 22$ instru $\% \mathrm{C} 3 \% \mathrm{~A} 7 \% \mathrm{C} 3 \% \mathrm{~A} 30 \% 20$ normativa $\%$ 20substratos \% 22. Acesso em: 26/09/2006.

GONCALVES, J.L.M. \& POGGIANI, F. Substratos para produção de mudas florestais. In: CONGRESSO LATINOAMERICANO DE CIÊNCIA DO SOLO, 13., Águas de Lindóia, 1996. Resumos. Piracicaba, Sociedade LatinoAmericana de Ciência do Solo, 1996. CD-ROM
GRUBER, Y.B.G. Otimização da lâmina de irrigação na produção clonais de eucalipto (Eucalyptus urophylla x Eucalyptus grandis var. Plathyphylla). Piracicaba, Escola Superior de Agricultura Luiz de Queiroz, 2006. 144p. (Tese de Mestrado)

HIGASHI, E.N.; SILVEIRA, R.L.V.A. \& GONÇALVES, A.N. Monitoramento nutricional e fertilização em macro, mini e microjardim clonal de Eucalyptus. In: GONÇALVES, J.L.M. \& BENEDETTI, V. Nutrição e fertilização florestal. Piracicaba, IPEF, 2000. p.192-215.

ISMAEL, J.J. Efeitos da fertilização nitrogenada e da umidade do substrato na aclimatação e na adaptação no campo de mudas de Eucalyptus grandis W. (Hill ex. Maiden). Jaboticabal, Universidade Estadual Paulista, 2001. 106p. (Tese de Doutorado)

KRÄMER, P.J. \&. BOYER, J.S. Water relations of plant and soils. San Diego, Academic Press, 1995. 495p.

LIMA, P.C.; BARROS, N.F.; NOVAIS, R.F. \& MOSQUIM, P.R. Alterações na absorção e distribuição de nutrientes minerais em plantas de Eucalyptus spp submetidas a deficiência hídrica no solo. In: CONFERÊNCIA IUFRO SOBRE SILVICULTURA E MELHORAMENTO DE EUCALIPTOS, 1997, Salvador. Anais. Salvador, Embrapa, 1997. p.38-45.

LOPES, J.L.W. Irrigação em viveiros de espécies florestais. In: CURSO DE PRODUÇÃO DE MUDAS FLORESTAIS META AMBIENTE. Resumos. Botucatu, 2006. CD-ROM

LOPES, J.L.W. Produção de mudas de Eucalyptus grandis W. (Hill ex. Maiden) em diferentes substratos e lâminas de irrigação. Botucatu, Universidade Estadual Paulista, 2004. 100p. (Tese de Mestrado)

LOPES, J.L.W.; GUERRINI, I.A.; SAAD, J.C.C. \& SILVA, M.R. Efeitos da irrigação na sobrevivência, transpiração e no teor relativo de água na folha em mudas de Eucalyptus grandis em diferentes substratos. Sci. Forestalis, 68:97106, 2005.

MALAVOLTA, E.; VITTI, G.C. \& OLIVEIRA, S.A. Avaliação do estado nutricional das plantas: Princípios e aplicações. 2.ed. Piracicaba, Potafós, 1997. 315p.

MARTINEZ, P.F. Manejo de substratos para horticultura. Campinas, Instituto Agronômico, 2002. p.53-76. (Documentos, 70)

SASSE, J.; SANDS, R.; WHITEHEAD, D. \& KELLIHER, F.M. Comparative responses of cuttings and seedlings of Eucalyptus grandis and Eucalyptus globulus to water stress. Tree Physiol., 16:287-294, 1996.

SCHMITZ, J.A.; SOUZA, P.V. \& KÄMPF, A.N. Propriedades químicas e físicas de substratos de origem mineral e orgânica para o cultivo de mudas em recipientes. Ci. Rural, 32:937-944, 2002.

SILVA, M.R. Efeitos do manejo hídrico e da aplicação de potássio na qualidade de mudas de Eucalyptus grandis W. (Hill ex. Maiden). Botucatu, Universidade Estadual Paulista, 2003. 100p. (Tese de Doutorado) 
SILVEIRA, R.L.V.A.; HIGASHI, E.N.; SGARBI, F. \& MUNIZ, M.R.A. Seja o doutor do seu eucalipto. Piracicaba, Potafós, 2001. 32p.
TRIGUEIRO, R.M. \& GUERRINI, I.A. Uso de biossólido como substrato para produção de mudas de eucalipto. Sci. Forestalis, 64:150-162, 2003. 